\title{
Epidemias, instituciones y Estado. La salud en Santiago de Chile, 1810-1842
}

\section{Epidemics, Institutions, and the State. Health in Santiago de Chile, 1810-1842}

\section{Epidemias, instituições e o Estado. A saúde em Santiago do Chile, 1810-1842}

Paula Caffarena Barcenilla, PhD $^{1}$

Recibido: 12 de diciembre de $2020 \cdot$ Aceptado: 15 de abril de 2021

doi: https://doi.org/10.12804/revistas.urosario.edu.co/revsalud/a.10595

Para citar este artículo: Caffarena Barcenilla P. Epidemias, instituciones y Estado: la salud en Santiago de Chile, 1810-1842. Rev Cienc Salud. 2021;19(Especial):1-18. https://doi.org/10.12804/revistas.urosario.edu.co/revsalud/a.10595

\section{Resumen}

Introducción: la pandemia de covid-19 ha suscitado un nuevo interés en la historia de la salud. Tanto en Chile como en otras partes del mundo, la búsqueda de respuestas en torno a cómo las sociedades pasadas han enfrentado la llegada de alguna epidemia ha hecho recordar que el contagio forma parte de nuestra historia y la estrecha relación que existe entre enfermedad y Estado. Desarrollo: se estudia la dimensión pública de la salud en Santiago de Chile entre los años 1810 y 1842, indagando, principalmente, en las respuestas que el Estado y sus instituciones entregaron en materia sanitaria frente a la aparición de brotes epidémicos. Se revisan las epidemias generadas por escarlatina, viruela y erisipela, dado que fueron las enfermedades que desencadenaron una respuesta de carácter institucional. Conclusión: la aparición de dichos brotes epidémicos se tradujo en que el Estado, a través de instituciones como el Protomedicato, la Junta de Sanidad, la Junta de Beneficencia o la Junta de Vacuna, articuló una respuesta sanitaria que se centró en mejorar las condiciones de higiene de la ciudad. A su vez, dio cuenta de dimensión pública de la enfermedad y de la capacidad que el Estado tuvo de poner en marcha medidas concretas que permitieran frenar los estragos de las epidemias.

Palabras clave: epidemias; Estado; instituciones sanitarias; higiene.

\section{Abstract}

Introduction: The covid-19 pandemic has generated a new interest in the history of health. In Chile and other parts of the world, search for answers regarding societies that have dealt with epidemics reminds us that contagions have been a part of our history and that there is a close relationship between disease and the State. Development: We studied the public dimension of health in Santiago de Chile between 1810 and 1842, mainly considering the responses of the State and its institutions toward epidemic outbreaks. In addition, we reviewed the scarlet fever, smallpox, and erysipelas epidemics, as these were the diseases that triggered an institutional response. Conclusion: The appearance of these epidemic outbreaks signified that the State, through institutions such as the Protomedicato, Junta de Sanidad, Junta de

1 CIDOc-Escuela de Historia, Universidad Finis Terrae (Chile). Correspondencia: pcaffarena@uft.cl ORCID: https://orcid.org/0000-0002-2609-6413. 
Beneficencia, or Junta de Vacuna, articulated a sanitary response that focused on improving the hygienic conditions of the city. At the same time, epidemic outbreaks also showed the public dimension of a disease and the State's capacity to implement specific measures to cope with the ravages of epidemics.

Keywords: epidemics; state; sanitary institution; hygiene.

Resumo

Introdução: a pandemia que vivemos de covid-19 despertou um novo interesse na história da saúde. Tanto no Chile como em outras partes do mundo, a busca por respostas sobre como as sociedades do passado enfrentaram a chegada de uma epidemia nos lembrou que o contágio faz parte da nossa história e da estreita relação que existe entre a doença e o Estado. Desenvolvimento: estuda-se a dimensão pública da saúde em Santiago do Chile entre os anos 1810 e 1842, investigando principalmente as respostas que o Estado e suas instituições deram em matéria de saúde ante o aparecimento de surtos epidêmicos. Revisamos as epidemias geradas pela escarlatina, varíola e erisipela, por serem as doenças que desencadearam uma resposta institucional. Conclusão: o surgimento desses surtos epidêmicos fez com que o Estado, por meio de instituições como o Protomedicato, o Conselho de Saúde, o Conselho de Caridade ou o Conselho de Vacinas, articulasse uma resposta sanitária centrada na melhoria das condições de higiene da cidade. Ao mesmo tempo, deu conta da dimensão pública da doença e da capacidade de que dispõe o Estado para implementar medidas concretas que permitiram conter a devastação das epidemias.

Palavras-chave: epidemias; Estado; instituições de saúde; higiene.

\section{Introducción}

a pandemia que vivimos de covid-19 ha suscitado un nuevo interés en la historia de la
salud. Tanto en Chile como en otras partes del mundo, la búsqueda de respuestas en torno a cómo las sociedades pasadas han enfrentado la llegada de alguna epidemia, nos ha recordado que el contagio forma parte de nuestra historia y, por tanto, tienen una larga data las restricciones sanitarias que hoy se implementan para frenar su avance. Como ha señalado George Duby, frente a brotes epidémicos, las ciudades, desde la Antigüedad, "se replegaban sobre sí mismas, evitaban a los extraños, pues se sospechaba que llevaban consigo la corrupción” (1, p. 90). A su vez, esta crisis ha puesto de relieve la importancia de contar con sistemas de salud pública sólidos, con recursos económicos y humanos suficientes, que garanticen a todos los ciudadanos el acceso a los tratamientos y cuidados necesarios.

¿Qué puede decirnos nuestro pasado sobre la emergencia sanitaria que vivimos en nuestro presente? La historia de la salud pública y la historia social de las enfermedades, nos permiten aventurar algunas respuestas, en la medida en que ambas perspectivas estudian las respuestas sociales e institucionales que se generaron en torno a la enfermedad, a la vez que nos muestran cómo en momentos de crisis sanitarias, el Estado adquiere un papel primordial(2). Considerando ambos enfoques, este estudio se pregunta por la dimensión pública 
de la salud en Santiago de Chile entre los años 1810 y 1842, indagando, principalmente, en las respuestas que el Estado y sus instituciones entregaron en materia sanitaria. ${ }^{2}$

La historia de la salud durante los primeros cuarenta años del siglo xix en Chile es la historia una sociedad habituada a la presencia de la enfermedad, pero no paralizada frente a ella. Conscientes de la incertidumbre y del peligro que generaba la llegada de alguna enfermedad, las autoridades de aquel periodo tomaron medidas para combatirlas y, así, frenar su avance. El periodo que nos interesa abordar corresponde a uno de gran convulsión política, social y económica. Se trata del momento en que Chile obtuvo la independencia de la monarquía hispana e inició un proceso de conformación estatal. Ello trajo consigo una gran precariedad fiscal, a la vez que una inestabilidad en las instituciones que comenzaban a formar la burocracia del Estado (3). Junto a ello, se trató de un periodo que vio cómo se intensificaba el proceso de urbanización que, a fines del siglo xix e inicios del siglo xx, se cristaliza en lo que conocemos como la cuestión social, que devela la profundidad de la crisis sanitaria que existía en los centros urbanos de la zona central de Chile (4). Este proceso de urbanización no puede ser soslayado, en la medida en que autores como Diego Armus y Roy Porter han podido establecer que "en la transmisión de las enfermedades, el proceso de urbanización tuvo un papel decisivo" (5, pp. 6 y 7).

Como bien nos han mostrado Rafael Sagredo, Enrique Laval y Ricardo Cruz Coke, muchas fueron las enfermedades que circularon en este periodo, entre las cuales la viruela, la disentería, el tifus, la erisipela, la influenza y la fiebre puerperal fueron las más recurrentes (7-9). Sin embargo, los documentos que se conservan en los Archivos Nacionales muestran que no todas estas enfermedades desencadenaron una respuesta de carácter institucional. Por ejemplo, si bien ocurrieron dos epidemias de fiebre puerperal, una en 1822 y otra en 1836, los registros que hasta la fecha hemos revisado no dan cuenta de una respuesta institucional similar a la que hubo frente a las epidemias de viruela, erisipela negra o escarlatina. Es probable que esta situación se vincule con la capacidad de propagación que tenían dichas enfermedades. La fiebre puerperal, por ejemplo, si bien podía tomar un carácter epidémico "atacando no solo a las mujeres paridas, sino a las que se hallan aun en cinta, a los niños, las personas del servicio y las enfermas de cirugía que contiene en ocasiones el mismo establecimiento" (10), se mantuvo concentrada en los hospitales. No así la viruela o la escarlatina, que tenían el potencial de avanzar por los diferentes centros urbanos del país.

¿Qué características tuvo el sistema sanitario de la época? ¿Cuáles fueron las instituciones que concentraron las funciones sanitarias? ¿Qué temas se discutieron frente a una epidemia?

2 El periodo que aborda este estudio se inicia en 1810, con los primeros pasos de Chile en cuanto república independiente, y finaliza en 1842, año en que se fundó la Facultad de Medicina de la Universidad de Chile. Consideramos que este fue un hito relevante en la medida en que se inició un nuevo proceso institucional en torno a la medicina, centralizando las decisiones, los estudios y, posteriormente, la enseñanza en la Universidad de Chile. 
¿Con qué recursos y herramientas contó el Estado para enfrentar los brotes epidémicos? Son algunas de las preguntas que nos interesa responder a lo largo de este estudio.

Nuestra hipótesis propone que durante los primeros cuarenta años del siglo xix existió un sistema sanitario compuesto por instituciones que buscaron dar respuesta a los problemas de salud que se comenzaban a identificar. Las epidemias, principalmente las provocadas por escarlatina, viruela y erisipela, fueron momentos en que dicha institucionalidad emitió respuestas o bien ejecutó acciones concretas en asuntos sanitarios, expresando la dimensión pública de la salud.

En términos metodológicos, para identificar y analizar la respuesta institucional frente a las epidemias ya mencionadas, fue necesario revisar los registros tanto de los fondos del Ministerio del Interior que alberga el Archivo Nacional de Santiago como los reportes que aparecieron en la prensa, particularmente La Aurora de Chile, El Mercurio de Chile y El Araucano. A través de ellos, fue posible acceder a los informes del Protomedicato, de la Junta de Vacuna y también a las disposiciones sanitarias que emitieron las autoridades frente a la aparición de alguna epidemia. Respecto a las defunciones que se producían a causa de estas, los trabajos realizados por Enrique Laval sobre el Hospital San Juan de Dios y el Hospital San Francisco de Borja, junto a algunos informes de prensa y fuentes impresas como el Anuario Estadístico, nos entregan algunas aproximaciones sobre el número de fallecidos a causa de las epidemias.

Este artículo se estructura en dos partes. La primera de ellas busca analizar el estado sanitario de la ciudad de Santiago durante 1810-1842, con el fin de comprender las principales enfermedades y epidemias que afectaron a la población, a la vez que busca identificar los principales problemas que se generaron en temas sanitarios. La segunda parte profundiza en torno a las instituciones que tuvieron a su cargo los temas sanitarios, analizando sus funciones y las respuestas concretas que entregaron frente a situaciones sanitarias específicas.

\section{Desarrollo}

\section{La situación sanitaria en Santiago: enfermedades y epidemias}

Desde fines del siglo xviI, el proceso de urbanización comenzó a intensificarse en la ciudad de Santiago. Se estima que su radio urbano se cuadruplicó entre dicho periodo y las dos primeras décadas del siglo xix, hasta alcanzar un total de 60000 habitantes (11). Esta situación repercutió de forma directa en las condiciones sanitarias de la ciudad, pues comenzó a evidenciarse la carencia de infraestructura urbana y que a mayor concentración de población, ciertas enfermedades se hacían más recurrentes o se agudizaban. Guillermo Blest, médico de origen 
irlandés que había llegado a Chile en 1824, constató, por ejemplo, que en las zonas rurales las enfermedades infectocontagiosas, como la viruela, la escarlatina o el tifus, afectaban en menor medida a la población, a diferencia de las zonas urbanas, que veían un aumento de estas. La prevalencia de las teorías miasmáticas puso la atención en la circulación del aire como sustento explicativo de la enfermedad; es por ello por lo que, en opinión de Blest, las mejores condiciones de salud que se observaban en las zonas rurales se debían a que allí se respiraba "libremente el aire atmosférico: ni aguas detenidas perturban con sus nocivos y pestíferos vapores el tranquilo y puro curso de su sangre. Tales son las delicias del campo, tales las causas que proporcionan salud y larga vida a sus habitantes” (12, pp. 14 y 15).

En los centros urbanos, particularmente en Santiago, el hacinamiento y las malas condiciones de vida aumentaron la recurrencia de la enfermedad. Lo primero que Blest destacó fue la mala infraestructura urbana que tenía la ciudad de Santiago, junto a lo mal empedradas en que se encontraban las calles. Llamó la atención sobre las malas condiciones en que estaban las acequias, pues se habían transformado en "receptáculos de toda clase de inmundicias, y no teniendo salida cómoda, mueren al derredor de la población en infeccionados charcos, eternos laboratorios de putrefacción” (12, p. 10).

Los problemas de higiene comenzaron a tomar la mayor importancia y no solo fueron reconocidos como causa o agravante de las epidemias que afectaron a la población, sino también como el origen de las precarias condiciones en que funcionaban los dos hospitales de la ciudad de Santiago: el Hospital San Juan de Dios (hSJD) y el Hospital San Francisco de Borja (нSғв), este último destinado a recibir mujeres en sus dependencias (13, pp. 10-13). ${ }^{3}$

Cada hospital contaba con alrededor de 100 camas que se copaban rápidamente, por lo que el alza en la demanda de estas, causada por el aumento de enfermos durante las epidemias y también por los heridos que dejaban los conflictos armados, presionó fuertemente al sistema, sin que este pudiera dar una respuesta adecuada (13). Los principales problemas que los hospitales tuvieron fueron la alta mortalidad, que fluctuó entre el $20 \%$ y el $27 \%$, y las malas condiciones de infraestructura con que los enfermos eran recibidos $(9,13) .{ }^{4} \mathrm{En}$ 1828, José Joaquín de Mora publicaba en el Mercurio Chileno que el estado de los hospitales era deplorable y que "las salas en invierno se encontraban tan abiertas que más bien parecen corredores al aire libre, páramos, que salas de hospital; almacenes mal cuidados que asilos del dolor; sin abrigo para el frío; los soldados enfermos envueltos en sus frazadas, sin ropa de hospital; sucios todos los enseres” (14).

3 Entre 1818 y 1828, las enfermas fueron enviadas a la Casa de Recogidas ubicada a los pies de Cerro Santa Lucía, ya que el HSFB se transformó en hospital militar. El HSJD había sido fundado en el siglo XVI; mientras que el HSFB había comenzado a funcionar en 1772, gracias a las gestiones del gobernador Antonio Guill y Gonzaga.

4 Entre 1807 y 1837, en el HSFB se registró una mortalidad promedio del 27.7\%; mientras que en el HSJD la cifra bordeaba el $20 \%$ para el mismo periodo. 
En el año 1814, por ejemplo, gracias a un recuento sobre morbilidad del HsJD, podemos ver que fallecieron un total de 1840 personas en dicho centro, entre ellas el $44 \%$ murió de enfermedades relacionadas con afecciones a la piel (sífilis, impétigos, sarna, eczema, entre otras); el $12 \%$, de chavalongo; el $8 \%$, de neumonías y pleuresías, y el $9 \%$, de viruela (figura 1).

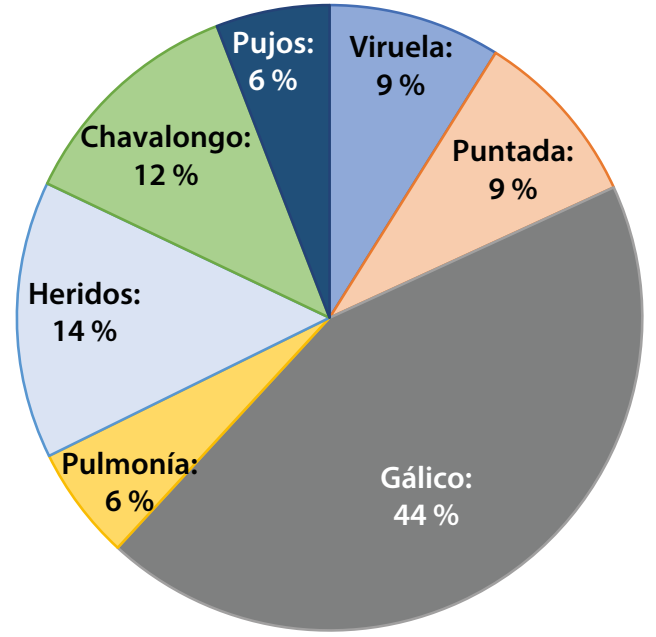

Figura 1. Estadística de morbilidad: Hospital San Juan de Dios, 1814

Fuente: tomado de (9).

Si bien estas cifras solo corresponden a un año y, además, a un momento político particular vinculado con el envío de tropas por parte del virrey Abascal para frenar el proceso revolucionario en Chile, lo que podría explicar el alto número de heridos que ingresó al hospital, nos permiten realizar algunas observaciones respecto a las enfermedades que afectaron a la población y al modo en que estas se identificaron.

La mayor cantidad de fallecidos que el hospital reportó fue a causa de gálico, lo cual no solo incluía a los enfermos de sífilis, sino también a todas las enfermedades que afectaban la piel. Lo mismo ocurrió con los fallecidos por puntadas, que incluyó neumonías y pleuresías. Otro caso similar fue el chavalongo o fiebre tifoidea, que se confundió con otras enfermedades como granulia, endocarditis séptica, otras septicemias y, principalmente, con el tifus exantemático (15).

Esto sugiere que la confusión diagnóstica fue un problema permanente con el que tuvieron que lidiar los médicos de la época, pues si bien a lo largo del siglo se fueron distinguiendo las diferentes afecciones, aun para los primeros cuarenta años del siglo xix, no todas las enfermedades estuvieron claramente identificadas. Es posible pensar que ello repercutió en la mortalidad registrada en los hospitales, pues muchos de los enfermos que llegaban no podían ser tratados de manera efectiva. En el cuerpo médico, compuesto tanto por nacionales como por extranjeros, todavía persistían las explicaciones asociadas a la teoría miasmática que, si bien se complementaron con nuevas teorías como la fisiologista (16), estaban lejos de poder combatir 
la enfermedad de manera efectiva. ${ }^{5}$ Por ejemplo, un reporte presentado por el нSғв respecto a los medicamentos suministrados en 1824, muestra que las enfermedades eran tratadas con mantequilla de cacao, piedra infernal, éter sulfúrico, aceite de linaza, ácido nítrico, opio, entre otros (13).

Durante los treinta años que este artículo estudia, hubo brotes epidémicos de viruela, erisipela, escarlatina, fiebre puerperal e influenza, además de otras enfermedades de carácter endémico que aquejaban a la población (tabla 1). De estas enfermedades, la viruela, la escarlatina y la erisipela poseían identificación diagnóstica, se les asociaba una sintomatología y poseían manifestaciones que permitieron a los médicos de la época individualizarlas.

Tabla 1. Registro de brotes epidémicos (Santiago)

\begin{tabular}{ll}
\hline \multicolumn{1}{c}{ Año } & \multicolumn{1}{c}{ Epidemia } \\
\hline $1811-1812$ & Viruela \\
1813 & Viruela \\
$1822-1823$ & Erisipela negra y fiebre puerperal \\
$1830-1831$ & Viruela \\
1831 & Influenza \\
1832 & Escarlatina, viruela, influenza \\
1839 & Viruela \\
1842 & Escarlatina \\
\hline
\end{tabular}

Fuente: elaborada a partir la información contenida en las fuentes y bibliografía citada a lo largo de este estudio.

En el caso de la viruela, el siglo xviI vio aparecer una serie de tratados médicos que permitieron clasificarla como una fiebre eruptiva, en que la aparición de pústulas cutáneas era el elemento más distintivo (17-19). Desde 1820, la escarlatina pudo ser diferenciada de la difteria por la presencia del característico exantema que venía acompañado de dolor de garganta,

5 Para el periodo que estudiamos, Chile contaba con un número reducido de médicos autorizados para ejercer la profesión. De acuerdo con la bibliografía revisada y citada a lo largo de este artículo, ejercían la medicina en Chile fray Rosauro Acuña, Eusebio Oliva, Pedro Morán e Isidro Zapata. Junto a ellos, una serie de médicos extranjeros comenzaron a desarrollar el oficio en el país, como el español Manuel Grajales, que se encontraba en Chile desde 1808; el inglés Nataniel Cox, que había llegado en 1814 a Santiago; Guillermo Blest, llegado en 1824; Lorenzo Sazié, contratado por el Gobierno de Chile en 1834; Francisco Lafargue, llegado a Chile en 1840; Carlos Buston, llegado a Chile en julio de 1823; Juan Miquel; José de Passamán; Tomás Leighton; entre otros. Estos médicos diagnosticaron a los enfermos y dieron cuenta de las enfermedades que aquejaban a la población. Produjeron también escritos que analizaron la situación sanitaria nacional, como fue el caso de las publicaciones de Guillermo Blest, Observaciones sobre el actual estado de la medicina en Chile con la propuesta de un pan para su mejoría (1826) y Ensayo sobre las causas más comunes y activas de las enfermedades que se padecen en Santiago de Chile con indicaciones de los mejores medios para evitar su destructora influencia (1828), y de Manuel Julián Grajales, Descripción de la verdadera, y falsa vacuna y modo de ingerir el fluido vacuno, con los accidentes que acaecen antes y después de su ingerción (1822). 
fiebre, inflamación de ganglios y abscesos en la garganta y amígdalas (20). La erisipela, por su parte, fue una enfermedad infecciosa que provocaba una inflamación cutánea en la piel, y si bien su etiología no quedó establecida hasta fines del siglo xix, se habían individualizado sus síntomas y manifestaciones (21).

El impacto demográfico que estas enfermedades tuvieron es difícil de establecer, pues los registros estadísticos para esta época son escasos y la mayor parte de tiempo, incompletos. ${ }^{6} \mathrm{De}$ la erisipela solo sabemos, por el testimonio que dejó Camilo Henríquez, en el Mercurio de Chile, citado en el libro de Pedro Lautaro Ferrer (22), que durante abril de 1822 se sepultaron 303 cadáveres afectados de dicha enfermedad. De la escarlatina, y si confiamos en los datos de las inhumaciones del Cementerio de Santiago que proporciona el Anuario Estadístico, es posible señalar que entre 1830 y 1832, las inhumaciones de niños asociadas a esta enfermedad aumentaron en un 53.1\%; mientras que para el mismo periodo la mortalidad en hombres aumentó un $36.6 \%$, y en el caso de las mujeres, un $39.4 \%$ (23). ${ }^{7}$

Para el caso de la viruela, contamos con una mayor cantidad de testimonios que indican que la letalidad de los enfermos que ingresaban a algún hospital fluctuó entre el $10 \%$ y el $30 \%$. Por ejemplo, para la epidemia que se desarrolló en Santiago en 1811 y continuó durante casi todo 1812, de los enfermos que ingresaron al HsjD, fallecieron cerca del $26 \%$. En 1813, una nueva epidemia afectó a la ciudad y la Aurora de Chile reportó que habían ingresado al HsjD 244 personas, de las cuales el 13\% había muerto a causa de dicha enfermedad (24). Las cifras se tornaron aún más alarmantes en la epidemia de viruela que afectó a la zona central de Chile en 1830, pues de acuerdo con lo indicado por el médico Thomas Leighton, al Hospital de Valparaíso ingresaron 242 enfermos de viruela, de los cuales falleció el 31 \% (tabla 2) (25).

Tabla 2. Razón de los apestados de viruelas que han entrado y muerto desde enero hasta el 1.․ de agosto de 1830

\begin{tabular}{lcc}
\hline & Entrados & Muertos \\
\hline Hombres & 106 & 34 \\
Mujeres & 136 & 43 \\
Total & 242 & 77 \\
\hline
\end{tabular}

Fuente: El Mercurio de Valparaíso, 5 de agosto de 1830.

Si bien los reportes poseen una naturaleza heterogénea que dificulta su comparación, podemos sugerir que las tres enfermedades señaladas tuvieron una elevada letalidad. Esto ocurrió en un escenario complejo, en términos sanitarios, pues el crecimiento demográfico

6 Solo a partir de 1843 Chile contó con una Oficina de Estadísticas.

7 El Anuario Estadístico, publicado en 1861, recoge datos desde 1830 hasta los datos publicados en el censo de 1858. Sus redactores explicaban que la información relativa a cementerios solo fue posible incorporar la de Santiago, pues los otros recintos no tenían registros y solo enviaron los del año 1858. 
que comenzó a vivir la ciudad de Santiago, sumado a la carencia de infraestructura sanitaria, dada tanto por la precariedad de los hospitales como por la insalubridad que presentaban los espacios públicos, desencadenó una respuesta de carácter institucional, cuyo objetivo fue frenar el avance de estas epidemias.

\section{Enfrentar las epidemias: respuesta estatal e institucionalidad sanitaria}

Durante los primeros cuarenta años del siglo xix, instituciones como el Protomedicato, la Junta de Sanidad, la Sociedad Médica, la Junta de Vacuna y la Junta Central de Beneficencia dieron vida al sistema sanitario de la época. A través de ellas, se diagnosticaron y enfrentaron los problemas de salud que afectaban a la población, particularmente aquellos vinculados con la higiene y los efectos de las epidemias. A estas instituciones recurrieron las autoridades del Gobierno para obtener información respecto al origen y a los tratamientos que se podían poner en marcha para afrontar la propagación de las epidemias.

La institución que mayor actividad mostró fue el Protomedicato que, a pesar de su origen colonial y de los cambios políticos de la época, se mantuvo en funcionamiento hasta fines del siglo xix. A pesar de ello, la emergencia que causó el recrudecimiento de enfermedades como la sífilis motivó la conformación de nuevas asociaciones sanitarias con objetivos más específicos, como fue la Comisión de Salud Pública, creada el 7 de agosto de 1813, cuyo principal fin fue "remediar los estragos del mal venéreo y se previno a todos los gremios, cuerpos, casas públicas y empleados del Estado que, con el aviso del Protomédico Ríos, franqueasen todos los auxilios necesarios para el cumplimiento de su comisión” (22, p. 458).

Frente a la epidemia de erisipela negra que atacó a la ciudad de Santiago en 1822, la primera acción que tomó el Gobierno, en ese momento a cargo de Bernardo O’Higgins, fue acudir al Protomedicato presidido por Eusebio Oliva, solicitando que

[...] se celebre una junta de los mejores facultativos en medicina [...], en la que se tratará de averiguar el origen de la introducción en el país de la erisipela negra gangrenosa, y de los medios más seguros que se pueden adoptar para evitar que se propague este mortífero contagio dándome cuenta sin perder momento de lo que dicha junta acordare en la materia. (26)

La junta fue presidida por el gobernador intendente de Santiago, José María Guzmán Ibáñez, quien tenía atribuciones de "policía de aseo, salubridad y seguridad" (27), y asistieron los médicos Manuel Grajales, Eusebio Oliva, Agustín Nataniel Cox y Bartolomé Coronilla, quienes, si bien indicaron que la epidemia aún se encontraba en una fase inicial, advirtieron del peligro si no se tomaban las medidas necesarias. Para ello, recomendaron "la limpieza general de las calles y casas de los particulares, conventos, cuarteles, acequias y muladares, 
[...] y el hospicio donde hay muchas aguas detenida y sin corrientes y llenas de substancias animales, vegetales y excrementos de todo viviente" $(28$, p. 122).

El Protomedicato identificó en la insalubridad de Santiago las causas de la propagación de la epidemia y la razón de por qué esta enfermedad afectaba más a los sectores pobres de la población. Haciendo referencias a la teoría miasmática, indicaron que el principal problema se encontraba en las habitaciones, que no eran más que "cuartos a la calle", y fueron consideradas unos "semillero de miasmas contagiosos":

Sus dueños duermen sin cama[,] la mayor parte entre inmundicias y humedades, y a las veces con carbón mal encendido, cuyos efectos de asfixia no han dejado de mostrarse algunas veces; $y$ así es que algunos profesores que asisten en los hospitales cívicos han visto que esta fiebre ha atacado con más fuerza y en mayor número a esta clase de enfermos que no a los pudientes que viven con aseo y limpieza. (26)

A pesar de que por falta de registros no podemos rastrear de manera detallada el curso de la epidemia, sabemos que en 1823 la erisipela continuó propagándose por Santiago. De acuerdo con un reporte del director supremo, Ramón Freire, esto se debía tanto a la ausencia "de un sistema curativo, como por el desaseo y falta de recursos en la clase miserable del pueblo" (28). Si bien las malas condiciones de higiene en Santiago tenían un componente estructural, la carencia de una infraestructura sanitaria acorde al crecimiento de la ciudad intensificaba los problemas. Ello motivó a Freire a destinar medidas generales para toda la población, como llamar a que, en los próximos ocho días bajo una multa de 12 pesos, todos los vecinos blanquearan sus casas, pues se reconocía en la cal un antídoto contra la erisipela. A su vez, estableció medidas específicas dirigidas a los sectores más pobres, por ejemplo, destinar una sala para hombres y otra para mujeres en el hsjD y obligar a los alcaldes a "conducir a la respectiva sala a las personas que en la comprensión de su barrio fueren atacadas de erisipela negra y carecieren de recursos para poner en práctica el plan curativo que se adoptase" (28).

La respuesta sanitaria del Gobierno se estructuró a partir de la información que entregó el Protomedicato, y las disposiciones que dictó Freire fueron una señal de cómo las epidemias dieron un impulso a la salud pública, a través de la implementación de nuevas medidas que buscaban mejorar las condiciones sanitarias de la población. La alarma que desde 1822 causó la epidemia de erisipela negra, junto a las precarias condiciones de higiene que los médicos destacaban, impulsó también la creación de la Junta de Sanidad (1822) que, presidida por Bernardo O’Higgins y compuesta por dos médicos, Manuel Julián Grajales y Agustín Nataniel Cox, buscó reunir allí todas las funciones sanitarias.

Si bien no tenemos noticias constantes de su desarrollo ni de las acciones concretas que llevó a cabo a lo largo de su funcionamiento, sabemos que el 29 de octubre de 1823 y, a propósito de la insalubridad del país, la junta publicó un documento que mencionó como causa 
de dicha insalubridad la "falta absoluta de la policía de aseo, especialmente en las acequias interiores" (29, f. 68). Como solución, planteó que los pobres debían tener "habitaciones sanas y baratas" y se debía "establecer una policía permanente, incorporándole por fondos los productos necesarios para limpieza interior de fosas y acequias, alumbrado y una pensión sobre carretas, carretores y carruajes de lujo que desempedran y arruinan las calles" (29).

La trayectoria que siguió la Junta de Sanidad en los años siguientes es incierta y dado el fallecimiento de algunos de sus miembros y por "haber concluido en otros el término de su nombramiento", su actividad disminuyó notoriamente, por lo que tres años después, el 15 de febrero de 1826, el Gobierno tuvo que decretar su restablecimiento (30).

Los vaivenes políticos y la necesidad de renovación que las autoridades del Gobierno manifestaron respecto a las instituciones provenientes del periodo colonial, se tradujeron en la supresión del Protomedicato en 1826 y, en su lugar, se estableció la Sociedad Médica. Al igual que el Protomedicato, tuvo una función consultiva pero se le concedió predominio en lo técnico. Entre sus funciones, estaba "proponer al gobierno, cuando fuere conveniente y lo ordene, facultativos para el servicio militar o de hospitales” o bien, en su artículo 10, se precisó que "las autoridades respetarán en la parte científica las decisiones de esta Sociedad, cuando sea requerida por el Gobierno para conocer de objetos de salud pública” (30, p. 407). En 1827, dicha sociedad fue remplazada por una Inspección General de Medicina; sin embargo, en menos de un año, la Sociedad Médica fue restablecida.

Nuevamente, los cambios políticos impactaron la institucionalidad sanitaria existente, de modo que la llegada del presidente Prieto, en 1830, y el anhelo por conformar un Estado centralizado, se tradujeron en la reapertura del Protomedicato, lo cual suscitó una gran controversia, pues, por su raíz colonial y porque ponía fin a la Sociedad Médica, fue fuertemente atacado a través del periódico satírico el Criticón Médico (8, p. 292). ${ }^{8}$ En este, y haciendo eco de la voz de los médicos, se referían al Protomedicato como el "envejecido y mohoso tribunal", cuyo código representaba "los siglos bárbaros del absolutismo y de la inquisición” (31, p. 434).

Si bien la reactivación del Protomedicato en la década de 1830 puede explicarse por la necesidad de centralizar la administración que buscó el Gobierno de ese entonces, ello no se tradujo en una clara delimitación de sus atribuciones (32). Por el contrario, en el artículo $2 .^{\circ}$ del decreto de su reapertura se indicó que el Protomedicato se restablecía "con todas sus atribuciones y reglamentos” (33, p. 121). Es decir, volvía a funcionar como el organismo responsable de organizar y supervisar el ejercicio de la medicina.

En términos generales, la acción del Gobierno frente a las epidemias mostró que a través de las instituciones sanitarias, como el Protomedicato, la Junta de Sanidad o la Sociedad

8 El Criticón Médico fue la primera publicación profesional médica de Chile. Se publicaron cuatro números entre el 5 y el 26 de junio de 1830, mediante los cuales se criticó la política de salud promovida por el Gobierno y por el Protomedicato presidido por Guillermo Blest. 
Médica, el Estado reguló e intervino en los asuntos de salud. Por ello, más allá de los cambios y cuestionamientos que dichas instituciones sufrieron en el periodo, su presencia dio cuenta de la relevancia que para las autoridades fue tomando la salud como un problema público. Las instituciones señaladas tuvieron como función atacar los males epidémicos, asesorar a las autoridades y uniformar la acción del Gobierno en materia de salud, aunque la práctica mostró que tuvieron una capacidad de acción limitada, ya fuese por falta de recursos o por la ausencia de seguimiento que se hacía de estas.

Así, frente a la aparición de casos de escarlatina en Valparaíso, en 1832, nuevamente el Protomedicato fue la institución llamada para identificar, diagnosticar y preparar un plan de acción frente a la enfermedad. El presidente Prieto solicitó al protomédico, el doctor Guillermo Blest, que se trasladara a Valparaíso e inspeccionara "prolijamente la enfermedad y fije de acuerdo con los médicos que hubieren en ese puerto el método curativo que debe observarse, y que V.S. de acuerdo con él adopte todas las medidas de precaución que exige el caso" (34, fj. 76). Esta función se replicó en 1842, cuando a raíz de los casos de escarlatina que aparecían en Santiago, el gobierno de Manuel Bulnes solicitó al Protomedicato un informe sobre los medios para combatir dicha enfermedad. El Protomedicato convocó a todos los profesores de medicina y redactó un completo reporte sobre la epidemia que aquejaba a la zona central del país. A través de este informe, el Protomedicato explicitó los diversos tratamientos que se podían utilizar. Sin embargo, frente a las preguntas que recibían respecto a cómo curar a los enfermos señalaron que era

[...] imposible detallar un método curativo en la fiebre escarlatina en general, respecto a que dicha enfermedad se presenta de diversos modos y que cada uno de ellos exige un tratamiento especial y diverso uno de otro, modificados estos por la edad, sexo, complicaciones con otras enfermedades, complexiones especiales del sujeto. (35, f. 7)

El 7 de abril de 1832, bajo la presión que generó la presencia de una epidemia de escarlatina que afectó a Valparaíso y avanzó hacia Santiago, fue creada la Junta Central de Beneficencia y Salud Pública, compuesta por doce miembros, cuyo único médico fue Guillermo Blest. Esta junta estuvo presidida por Manuel Blanco Encalada y debía establecer una vigilancia en educación, salud, trabajo, población y vivienda. Para cumplir estas tareas formó cinco comisiones: de educación y culto; de cementerios y hospitales; de cárceles, cuarteles y conventos; de policía de salubridad, comodidad y ornato, y de agricultura y comercio. Sus funciones iban a ser desarrolladas a nivel local por juntas provinciales y municipalidades (8). A pesar de este nuevo impulso, la Junta de Beneficencia y Salud Pública tuvo una vida precaria, pues muchas fueron sus atribuciones, lo que dificultó la ejecución de cualquier iniciativa (13).

La necesidad que el Gobierno tenía de enfrentar de manera más efectiva los problemas sanitarios se expresó en la Constitución de 1833, que trasladó la responsabilidad de velar por 
la sanidad a las municipalidades. Estas debían "cuidar de la policía de salubridad, comodidad, ornato y recreo" (36, p. 38). A pesar de que la medida buscaba centralizar la ejecución de las disposiciones sanitarias en una institución local, esto no se cumplió adecuadamente. Así lo expresó el presidente Manuel Bulnes, cuando a raíz del mal estado en que se encontraba la policía de aseo de Santiago, señaló que esto se debía a "lo defectuoso del sistema que hoy se emplea para atender a un ramo ligado estrechamente con la salubridad pública” (34, fj. 98).

La articulación que la medida anterior requería entre instituciones, como el Protomedicato y las municipalidades, sumado a la baja capacidad de ejecución que demostraron las propias municipalidades, fueron fiel reflejo de los problemas que tuvo el proceso de conformación institucional que caracterizó a la época, pero, a pesar de ello, dichas instituciones plasmaron la dimensión pública que tenía la enfermedad.

Tanto en el caso de la epidemia de erisipela como la de escarlatina, el Protomedicato ocupó un papel importante, en cuanto fue la institución que participó en el diagnóstico y en la elaboración de las medidas que se tomaron para combatir dichas epidemias. Sus intervenciones permitieron dar respuesta en torno al origen de las epidemias y también diseñar un plan con miras a detener su propagación. En los dos casos mencionados se propuso implementar medidas que permitieran mejorar la limpieza de la ciudad, pues la insalubridad de Santiago fue vista tanto como un foco de origen de las epidemias como un factor que las agudizaba.

Las epidemias de viruela difieren de lo señalado, pues esta enfermedad se podía combatir a través de la vacuna, y ello se tradujo en una respuesta institucional diferente. La vacunación se aplicaba en Chile desde 1805 y la institución a cargo de su difusión fuela Junta de Vacuna (37). Esta institución se formó en Chile en 1808, con la llegada al país del médico español Manuel Julián Grajales; luego, en 1812, un nuevo decreto reactivó la Junta de Vacuna, pero los avatares del proceso de independencia impidieron su consolidación. En 1830, un nuevo decreto reorganizó la Junta de Vacuna y, a diferencia de las juntas precedentes, contó con una mayor organización. Esto permitió expandir la cobertura de la vacuna y también que el Estado tomara mayor control sobre ella. En su reglamento, por ejemplo, se estableció que sus integrantes serían nombrados por el Gobierno y se exigió un registro de todas las personas vacunadas que indicara nombre, edad y sexo, el que debía ser entregado al Gobierno de modo trimestral (38, f. 200). Adicionalmente, se establecieron sueldos fijos para los vacunadores de la capital y de las provincias, así como un monto para solventar los gastos administrativos de la institución.

A pesar de ello, el número de personas que accedía a la vacuna era bajo, por lo que la viruela continuó siendo una amenaza recurrente para la población. En 1828, por ejemplo, una epidemia de viruela afectó a la zona central de Chile, ocasión en que las autoridades del Gobierno solicitaron a la Junta de Vacuna dar mayor impulso a la vacunación con el objetivo de aumentar su cobertura. Se les pidió, por ejemplo, enviar nuevos vacunadores 
a las zonas afectadas de viruela, para que pudieran difundir allí la vacuna y así frenar los contagios $(39,40)$.

El impulso que se buscó dar a la vacunación a través de esta junta fue insuficiente y el Gobierno procuró la forma de persuadir a la población para que acudiera a ser vacunada. Por ejemplo, en una circular enviada al obispo de Concepción en 1830, el Gobierno solicitó que los curas se involucraran directamente en la promoción de la vacunación, pues a su juicio la ayuda que estos pudieran prestar era uno de los medios más eficaces para propagar la vacuna. Se les solicitó que en sus "respectivas feligresías trabajen en cuanto esté de su parte por inclinar a los habitantes a que pasen por esta operación tan provechosa como fácil” (41, f. 96).

El ejemplo expuesto ilustra que la respuesta para enfrentar la viruela se articuló en torno a la necesidad de aumentar el número de personas vacunadas. En la búsqueda de este objetivo, se evidenciaron dos dificultades asociadas a la práctica de la vacunación: por una parte, se requería contar con un mayor número de vacunadores que recorrieran distintos lugar del país; por otra, también era indispensable que la población asistiera a los lugares donde se estaba vacunando. Así, la resistencia de la población que aún no acudía de manera voluntaria a recibir la vacuna fue un obstáculo en la difusión de esta y, como hemos visto, el Gobierno buscó el modo de revertir la situación.

\section{Conclusiones}

$\mathrm{D}$ urante los primeros cuarenta años del siglo xix, la ciudad de Santiago sufrió la llegada de una serie de epidemias que impulsaron la intervención de las autoridades del Gobierno en asuntos sanitarios. Ello se tradujo en que el Estado, a través de instituciones como el Protomedicato, la Junta de Sanidad, la Junta de Beneficencia o la Junta de Vacuna, articuló una respuesta sanitaria. En este sentido, los brotes epidémicos de viruela, escarlatina y erisipela negra que ocurrieron entre 1810 y 1842 nos han permitido analizar dicha respuesta e identificar las principales medidas que se implementaron.

Tanto para la escarlatina como para la erisipela, las medidas sanitarias que se promovieron buscaron mejorar las condiciones de higiene de la ciudad, pues en la insalubridad de Santiago y, en concordancia con la teoría miasmática, se situó el origen de los males epidémicos que los afectaban. En este sentido, podemos sugerir que ya desde inicios del siglo xix, el higienismo influyó en las políticas de control de las enfermedades. Son ejemplos de lo anterior el recurrente llamado que médicos como Guillermo Blest hicieron respecto a la suciedad de las acequias o el llamado a mejorar las condiciones habitacionales de la población.

El caso de la viruela evidenció una respuesta institucional diferente. Al existir una vacuna que había demostrado ser efectiva, el Estado se concentró en mejorar la difusión de esta. Para 
ello, la Junta de Vacuna fue la institución que permitió articular los esfuerzos para mejorar la difusión y también para generar mayor registro respecto al número de vacunados. A pesar de ello, la viruela siguió generando brotes epidémicos de importancia y solo hacia 1918, con el decreto que hizo de la vacunación un acto obligatorio, se consiguió la reducción de casos y, finalmente, hacia 1980, la erradicación definitiva de la enfermedad en el mundo.

Las instituciones que aquí hemos estudiado entregan luces sobre las características que tuvo el sistema sanitario de la época. Por una parte, mostró ser dependiente de los cambios políticos del periodo, lo cual se tradujo en la escasa proyección de dichas instituciones. Por ejemplo, solo en el Protomedicato o en la Junta de Vacuna es posible identificar una acción continua en el tiempo, pues otras instituciones, como la Junta de Sanidad o la Sociedad Médica, tuvieron una corta vida. Por otra, el sistema sanitario mostró dificultades para poner en marcha acciones concretas que permitieran frenar el avance de las epidemias. El caso de la escarlatina nos entrega un ejemplo sobre esto, pues los médicos reunidos al llamado del Protomedicato no pudieron indicar medidas generales para frenar el avance de la enfermedad, puesto que las particularidades de cada sujeto y su reacción frente a la epidemia impedían ese tipo de aproximación.

A pesar de lo anterior, este estudio ofrece un ejemplo de la estrecha relación que existe entre enfermedad, Estado y las instituciones de corte sanitario que dicho Estado es capaz de proveer. En dicha relación se materializa la dimensión pública de la enfermedad y se evidencia la capacidad de poner en marcha medidas concretas que frenen los estragos de las epidemias.

Partíamos en este artículo por preguntarnos qué nos puede enseñar nuestro pasado de lo que vivimos en nuestro presente. Probablemente y, a la luz del análisis que aquí hemos presentado, sea necesario considerar que cada epidemia debe pensarse en su propio contexto y con sus propias circunstancias. Al siglo xix correspondió una forma de entender la enfermedad y una serie de recursos y posibilidades para enfrentarla. En este sentido, mirar nuestro pasado no nos entregará las soluciones que nuestro presente requiere, pero sí nos permitirá comprender, con mayor profundidad, el impacto que causaron las epidemias, las dimensiones que se vieron trastocadas y también los obstáculos que se debieron enfrentar. Puede que allí encontremos algunas directrices para nuestro presente.

\section{Financiación}

F ste artículo forma parte del Proyecto Fondecyt de Iniciación n. ${ }^{\circ}$ 11170571, patrocinado Epor la Agencia Nacional de Investigación y Desarrollo de Chile. 


\section{Descargos de responsabilidad}
T a autora asume la responsabilidad de lo publicado y la Revista de Ciencias de la Salud no Lasume ninguna responsabilidad por ello.

\section{Conflicto de intereses}

Ninguno declarado.

\section{Referencias}

1. Duby G. Año 1000, año 2000: la huella de nuestros miedos. Santiago: Andrés Bello; 1995.

2. Cueto M, Palmer S. Medicine and public health in Latin America: a history. New York: Cambridge University Press; 2015.

3. López E. El proceso de construcción estatal en Chile: hacienda pública y burocracia (1817-1860). Santiago: DIBAM/Centro de Investigaciones Diego Barros Arana; 2014.

4. Illanes MA. En el nombre del pueblo, del estado y de la ciencia, (...): historia asocial de la salud pública, Chile, 1880-1973: hacia una historia social del siglo xx. Santiago: Colectivo de Atención Primaria; 1993.

5. Porter R. Blood and guts: a short history of medicine. London: Penguin Books; 2003.

6. Armus D. La ciudad impura: salud, tuberculosis y cultura en Buenos Aires, 1870-1950. Buenos Aires: Edhasa; 2007.

7. Sagredo R, Gazmuri C. Historia de la vida privada en Chile: el Chile moderno. De 1840 a 1925. Santiago: Penguin Random House; 2005.

8. Cruz Coke R. Historia de la medicina chilena. Santiago: Andrés Bello; 1995.

9. Laval E. Historia del Hospital San Juan de Dios: apuntes. Santiago: Asociación Chilena de Asistencia Social; 1949.

10. Zorrilla P. Medicina. Consideraciones sobre la fiebre puerperal: memoria de prueba de don Pablo Zorrilla en su examen para optar al grado de Licenciado en Medicina, leída a fines de marzo de 1862. Anales de la Universidad de Chile (Santiago). 1862;tomo 20(serie 1).

11. Ramón A de. Santiago de Chile: historia de una sociedad urbana. Santiago: Catalonia; 2007.

12. Blest G. Ensayo sobre las causas más comunes y activas de las enfermedades que se padecen en Santiago de Chile con indicaciones de los mejores medios para evitar su destructora influencia. Santiago: Imprenta de R. Rengifo; 1828. 
13. Laval E. Historia del Hospital San Francisco de Borja de Santiago (1772-1944). Santiago: Ediciones Universidad Católica; 2011.

14. Mora JJ de. El Mercurio de Chile; 1828.

15. Laval E. Algunas notas sobre el desarrollo histórico de la fiebre tifoidea en Chile. Rev Chil Infectol [internet]. 2017 oct [citado 2020 dic 06];34(5):491-3. Disponible en: https:// scielo.conicyt.cl/scielo.php?script=sci_arttext\&pid=S0716-10182017000500491\&lng=es

16. Caffarena Barcenilla P. La enfermedad y sus causas en el siglo xix chileno: la mirada del doctor Guillermo Blest. Rev Chil Infectol. 2020 nov;37(5):591-6. https://doi.org/10.4067/ S0716-10182020000500591

17. O’Scanlan T. Práctica moderna de la inoculación con varias observaciones y reflexiones fundadas en ella, precedidas de un discurso sobre la utilidad de esta operación, y un compendio histórico de su origen y de su estado actual, particularmente en España, con un catálogo de algunos inoculados. Madrid: Hilario Santos; 1784.

18. Gil F. Disertación físico Médica en la cual se prescribe un método seguro para preservar a los pueblos de viruelas hasta lograr la completa extinción de ellas en todo el reino. Madrid: Impreso por don Joachim Ibarra; 1784.

19. Buchan G. Tratado de medicina doméstica. Madrid: Imprenta Real; 1792.

20. Swedlund A. Scarlet fever epidemics of the nineteenth century: a case of evolved pathogenic virulence? En: Herring DA, Swedlund AC, editores. Human biologists in the archives: demography, health, nutrition and genetics in historical populations. Cambridge: Cambridge University Press; 2002.

21. Enrique L. Las epidemias de erisipela en Chile. Rev Chil Infectol [internet]. 2011 ab [citado 2020 nov 24];28(2):179-80. Disponible en: https://scielo.conicyt.cl/scielo.php?scrip$\mathrm{t}=$ sci_arttext\&pid=S0716-10182011000200012\&lng=es

22. Lautaro Ferrer P. Historia general de la medicina en Chile. Tomo I. Santiago: Imp. Talca de J. Martín Garrido C.; 1904.

23. Anuario Estadístico de la República de Chile. Entrega segunda. Santiago: Imprenta Nacional; 1861.

24. Aurora de Chile, 31 de diciembre de 1813. Tomo 2, núm. 1. Santiago: Imprenta de Gobierno; 1813.

25. Estadística de viruela. El Mercurio de Valparaíso, 5 de agosto de 1830.

26. Informe firmado por los médicos Manuel Grajales, Eusebio Oliva, Agustín Nataniel Cox y Bartolomé Coronilla. Erisipela gangrenosa. El Mercurio de Chile, 3 de julio de 1822.

27. Enríquez L. Las intendencias y el gobierno interior de Chile entre 1810 y 1833 . Caravelle. 2017;109:161-78. https://doi.org/10.4000/caravelle.2505

28. Boletín de Leyes y Decretos del Gobierno. Santiago de Chile; mayo de 1823.

29. Documento de la Junta de Sanidad a propósito de la insalubridad extraordinaria del país. Archivo Nacional, Fondo Ministerio del Interior. Vol. 35; 1823.

30. Boletín de las Leyes y de las Órdenes y Decretos del Gobierno. Santiago: La Independencia; 1839. 
31. Barros Arana D. Historia general de Chile. Tomo 15. Santiago: Editorial Universitaria; 1999.

32. Piwonka G. El Protomedicato Republicano 1819 y 1842. Anales Chilenos de Historia de la Medicina (Santiago). 2006;16.

33. Lira JB. La lejislacion chilena no codificada, o sea Coleccion de leyes i decretos vijentes i de interes jeneral ordenada. Vols. 1-4. Santiago: "El Correo” de R. Varela; 1879.

34. Informe del presidente de la República Manuel Bulnes, 12 de septiembre de 1842. Archivo Nacional, Fondo Ministerio del Interior. Vol. 164.

35. Informe del presidente del Protomedicato Nataniel Cox, 31 de octubre de 1842, ANFMI, vol. 139, f. 7.

36. Constitución de la República de Chile. Jurada y Promulgada el 25 de mayo de 1833, Santiago: Imprenta de la Opinión; 1833.

37. Caffarena Barcenilla P. Viruela y vacuna: difusión y circulación de una práctica médica. Chile en el contexto hispanoamericano 1780-1830. Santiago: Editorial Universitaria; 2016.

38. Reglamento Junta de Vacuna. Archivo Nacional, Fondo Ministerio del Interior. Vol. 272; junio de 1830.

39. Circular dirigida a los intendentes de Santiago, Concepción y Maule y a los Gobernadores de Curicó, Talca y San Fernando. Archivo Nacional, Fondo Ministerio del Interior. Vol. 93; 1828.

40. Caffarena Barcenilla P. Una profesión sanitaria en la lucha contra la viruela: vacunadores en Chile, 1805-1887. Historia 396. 2020;10(núm. esp.):39-66.

41. Circular dirigida al Obispo/Diócesis de Concepción. Archivo Nacional, Ministerio del Interior. Vol. 93; 1830. 